Envel opes of $f$ amil i es of Legendre mappi ngs i $n$ the uni t tangent bundl e over the Eucl i dean space

\begin{tabular}{|l|l|}
\hline 著者 & TAKAHASH Nasat ond \\
\hline $\begin{array}{l}\text { j our nal or } \\
\text { publ i cat i on t i t l e }\end{array}$ & $\begin{array}{l}\text { Jour nal of Nat henat i cal Anal ysi s and } \\
\text { Appl i cat i ons }\end{array}$ \\
\hline vol une & 473 \\
\hline number & 1 \\
\hline page range & $408-420$ \\
\hline year & 2019-05- 01 \\
\hline URL & ht t p: //hdl . handl e. net /10258/00009919 \\
\hline
\end{tabular}




\title{
Envelopes of families of Legendre mappings in the unit tangent bundle over the Euclidean space
}

\author{
Masatomo Takahashi \\ Dedicated to Professor Takashi Nishimura on the occasion of his 60th birthday \\ November 7, 2018

\begin{abstract}
For families of hypersurfaces with singular points, a classical definition of an envelope is vague. In order to define an envelope for a family of hypersurfaces with singular points, we consider $r$-parameter families of frontals and of Legendre mappings in the unit tangent bundle over the Euclidean space. We define an envelope for the $r$-parameter family of Legendre mappings. Then the envelope is also a frontal. We investigate properties of the envelopes. As an application, we give a condition that the projection of a singular solution of a first order partial differential equation is an envelope.
\end{abstract}

\section{Introduction}

Envelopes are classical object in the differential geometry. There are a lot of applications of envelopes to differential geometry, differential equations and physics, for instance $[4,5,7,8$, $9,12,15,16,18,21,23]$. An envelope of a family of surfaces is a surface that is "tangent" to each member of the family at some point. If the surfaces are regular, then the tangent is well-defined. However, a definition of an envelope is vague for singular surfaces (surfaces with singular points). In [22], a definition and properties of an envelope for a one-parameter family of Legendre curves in the unit tangent bundle over $\mathbb{R}^{2}$ were given. In this paper, we clarify a definition of an envelope for a family of singular surfaces. As singular surfaces, we consider frontals and Legendre mappings in the unit tangent bundle over $\mathbb{R}^{n+1}$. The basic results on the singularity theory see $[1,2,4,13,14,17]$.

We consider $r$-parameter families of Legendre mappings and define an envelope in $\S 3$. Then the envelope of an $r$-parameter family of Legendre mappings is also a frontal. We give a necessary and sufficient condition that the $r$-parameter family of Legendre mappings has an envelope, see Theorem 3.6 as the envelope theorem. Moreover, we give relations between envelopes of a classical definition and of a family of Legendre mappings. As an application,

2010 Mathematics Subject classification: 58K05, 53A07, 57R45

Key Words and Phrases. envelope, family of Legendre mappings, frontal, singular solution. 
we give a condition that the projection of a singular solution of a first order partial differential equation is an envelope by using the envelope theorem in $\S 4$.

All maps and manifolds considered here are differentiable of class $C^{\infty}$.

Acknowledgement. The author would like to thank Professor Takashi Nishimura for his constant encouragements. The author would also like to thank Professor Kentaro Saji for valuable discussions and the referee for useful comments that improved the original manuscript. This work was a partially supported by JSPS KAKENHI Grant Number JP 17K05238.

\section{Preliminaries}

Let $\mathbb{R}^{n+1}$ be the $(n+1)$-dimensional Euclidean space with the inner product $x \cdot y=x_{1} y_{1}+$ $\cdots+x_{n+1} y_{n+1}$, where $x=\left(x_{1}, \ldots, x_{n+1}\right), y=\left(y_{1}, \ldots, y_{n+1}\right) \in \mathbb{R}^{n+1}$. The norm of $x \in \mathbb{R}^{n+1}$ is given by $|x|=\sqrt{x \cdot x}$.

Let $F: W \times \Lambda \rightarrow \mathbb{R}$ be an $r$-parameter family of smooth functions, where $W$ and $\Lambda$ are domains in $\mathbb{R}^{n+1}$ and in $\mathbb{R}^{r}$, respectively. Then one of the classical definition of an envelope $E_{I}$ is as follows, see for instance $[3,4,11]$ :

Definition 2.1 The envelope of the family $F$ is the discriminant set of $F$, that is, the set of points given by

$$
E_{I}=\left\{x \in \mathbb{R}^{n+1} \mid \text { for some } \lambda \in \Lambda, F(x, \lambda)=F_{\lambda_{j}}(x, \lambda)=0, j=1, \ldots, r\right\} .
$$

If $F(x, \lambda)=F_{\lambda_{j}}(x, \lambda)=0, j=1, \ldots, r$, we say that $x \in E_{I}$ with respect to $\lambda=\left(\lambda_{1}, \ldots, \lambda_{r}\right)$. Here $F_{\lambda_{j}}(x, \lambda)=\left(\partial F / \partial \lambda_{j}\right)(x, \lambda)$.

Example 2.2 Let $F: \mathbb{R}^{3} \times \mathbb{R} \rightarrow \mathbb{R}, F(x, y, z, \lambda)=(x-\lambda)^{3}-y^{2}$. Then $F=0$ is the image of the cuspidal edge for each fixed $\lambda \in \mathbb{R}$, see Figure 1 and Example 3.8. The definition and properties of cuspidal edges see [10, 20]. Since $F_{\lambda}(x, y, z, \lambda)=-3(x-\lambda)^{2}$, the envelope of the family $F$ is given by $E_{I}=\{(\lambda, 0, z)\}=x z$-plane.

Example 2.3 Let $F: \mathbb{R}^{3} \times \mathbb{R} \rightarrow \mathbb{R}, F(x, y, z, \lambda)=x^{3}-(y-\lambda)^{2}$. Then $F=0$ is the image of the cuspidal edge for each fixed $\lambda \in \mathbb{R}$, see Figure 2 and Example 3.9. Since $F_{\lambda}(x, y, z, \lambda)=2(y-\lambda)$, the envelope of the family $F$ is given by $E_{I}=\{(0, \lambda, z)\}=y z$-plane.

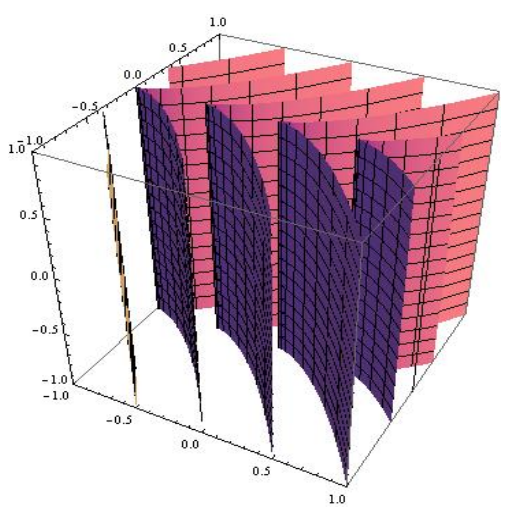

Figure 1.

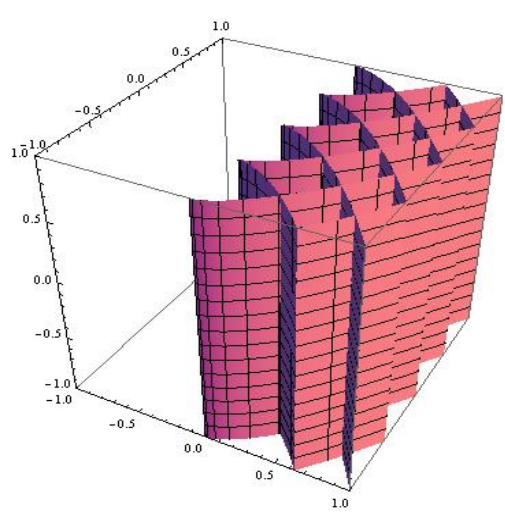

Figure 2. 
However, in the sense of the limit tangent plane of the cuspidal edge, $y z$-plane is not tangent to the cuspidal edge. Therefore, we would like to distinguish as envelopes, see Examples 3.8 and 3.9 .

Let $U \subset \mathbb{R}^{n}$ be a domain in $\mathbb{R}^{n}$. We say that $(f, \nu): U \rightarrow \mathbb{R}^{n+1} \times S^{n}$ is a Legendre mapping if $(f, \nu)^{*} \theta=0$, where $\theta$ is a canonical contact form on the unit tangent bundle $T_{1} \mathbb{R}^{n+1}=\mathbb{R}^{n+1} \times S^{n}$ over $\mathbb{R}^{n+1}$ (cf. $[1,2]$ ). Moreover, $f: U \rightarrow \mathbb{R}^{n+1}$ is a frontal (respectively, a front) if there exists a smooth mapping $\nu: U \rightarrow S^{n}$ such that $(f, \nu)$ is a Legendre mapping (respectively, a Legendre immersion). The condition $(f, \nu)^{*} \theta=0$ is equivalent to $d f(u) \cdot \nu(u)=0$ for all $u \in U$. If we denote $f(u)=\left(f_{1}(u), \ldots, f_{n+1}(u)\right), \nu(u)=\left(\nu_{1}(u), \ldots, \nu_{n+1}(u)\right)$ and $u=\left(u_{1}, \ldots, u_{n}\right)$, then the condition $d f(u) \cdot \nu(u)=0$ for all $u \in U$ is equivalent to

$$
f_{u_{i}}(u) \cdot \nu(u)=f_{1 u_{i}}(u) \nu_{1}(u)+\cdots+f_{n+1 u_{i}}(u) \nu_{n+1}(u)=0
$$

for all $u \in U$ and $i=1, \ldots, n$.

The parallel of a Legendre mapping $(f, \nu): U \rightarrow \mathbb{R}^{n+1} \times S^{n}$ is defined by $f^{k}: U \rightarrow$ $\mathbb{R}^{n+1}, f^{k}(u)=f(u)+k \nu(u)$, where $k \in \mathbb{R}$. Then it is easy to see that $\left(f^{k}, \nu\right): U \rightarrow \mathbb{R}^{n+1} \times S^{n}$ is also a Legendre mapping for each fixed $k \in \mathbb{R}$.

\section{Envelopes of families of Legendre mappings}

We say that $(f, \nu): U \times \Lambda \rightarrow \mathbb{R}^{n+1} \times S^{n}$ is an $r$-parameter family of Legendre mapping if $(f(\cdot, \lambda), \nu(\cdot, \lambda)): U \rightarrow \mathbb{R}^{n+1} \times S^{n}$ is a Legendre mapping for each $\lambda \in \Lambda \subset \mathbb{R}^{r}$.

Let $(f, \nu): U \times \Lambda \rightarrow \mathbb{R}^{n+1} \times S^{n}$ be an $r$-parameter family of Legendre mappings. Let $V \subset \mathbb{R}^{n}$ be an open subset and $e: V \rightarrow U \times \Lambda, e(p)=(u(p), \lambda(p))$ be a smooth mapping. We denote $E=f \circ e: V \rightarrow \mathbb{R}^{n+1}$.

Definition 3.1 We call $E$ an envelope (and e a pre-envelope) for the $r$-parameter family of Legendre mappings $(f, \nu)$, when the following conditions are satisfied.

(i) The set of regular points of $\lambda: V^{n} \rightarrow \Lambda^{r}$ is dense in $V$. (The Variability Condition.)

(ii) For all $p \in V$ and $i=1, \ldots, n, E_{p_{i}}(p) \cdot \nu(e(p))=0$. (The Tangency Condition.)

The definition of the envelope is a generalisation of the definition of the envelope of a one-parameter family of Legendre curves in [22]. By definition, we have the following.

Proposition 3.2 Let $(f, \nu): U \times \Lambda \rightarrow \mathbb{R}^{n+1} \times S^{n}$ be an r-parameter family of Legendre mappings. Suppose that $e: V \rightarrow U \times \Lambda$ is a pre-envelope and $E=f \circ e: V \rightarrow \mathbb{R}^{n+1}$ is an envelope of $(f, \nu)$. Then $E$ is a frontal. More precisely, $(E, \nu \circ e): V \rightarrow \mathbb{R}^{n+1} \times S^{n}$ is a Legendre mapping.

Proof. Since the tangency condition, we have $E_{p_{i}}(p) \cdot \nu(e(p))=0$ for all $p \in V$. It follows that $d E(p) \cdot(\nu \circ e)(p)=0$ for all $p \in V$. That is, $(E, \nu \circ e): V \rightarrow \mathbb{R}^{n+1} \times S^{n}$ is a Legendre mapping.

Proposition 3.3 Let $(f, \nu): U \times \Lambda \rightarrow \mathbb{R}^{n+1} \times S^{n}$ be an r-parameter family of Legendre mappings. Suppose that $e: V \rightarrow U \times \Lambda$ is a pre-envelope and $E=f \circ e$ is an envelope of $(f, \nu)$. Then we have the following. 
(1) $e: V \rightarrow U \times \Lambda$ is also a pre-envelope of $(f,-\nu)$ and $E=f \circ e$ is also an envelope of $(f,-\nu)$.

(2) $e: V \rightarrow U \times \Lambda$ is also a pre-envelope of $(-f, \nu)$ and $-E=-f \circ e$ is an envelope of $(-f, \nu)$.

Proof. (1) By definition, $(f,-\nu)$ is also an $r$-parameter family of Legendre mappings. Since $e$ is a pre-envelope of $(f, \nu), E_{p_{i}}(p) \cdot\left(-\nu(e(p))=-E_{p_{i}}(p) \cdot \nu(e(p))=0\right.$ for all $p \in V$. Hence, $e$ is also a pre-envelope and $E=f \circ e$ is also an envelope of $(f,-\nu)$.

(2) By similarly, we have the result.

Remark 3.4 By Proposition 3.3 (1), we may define an envelope for an $r$-parameter family of Legendre mapping in $P T^{*} \mathbb{R}^{n+1}$.

Remark 3.5 As the same definition, we can define an envelope of a family of Legendre mappings in the unit tangent bundle over a smooth manifold. Especially, we can define envelopes not only of families of Legendre mappings in the unit spherical bundle (cf. [19]), but also of families of frontals in the hyperbolic or de-Sitter space (cf. [6]).

We give a necessary and sufficient condition that the $r$-parameter family of Legendre mappings has an envelope. We call this result the envelope theorem (cf. [11, 22]).

Theorem 3.6 (The Envelope Theorem) Let $(f, \nu): U \times \Lambda \rightarrow \mathbb{R}^{n+1} \times S^{n}$ be an r-parameter family of Legendre mappings, and let $e: V \rightarrow U \times \Lambda$ be a smooth mapping satisfying the variability condition. Suppose that $n \geq r$. Then $e$ is a pre-envelope of $(f, \nu)$ (and $E$ is an envelope) if and only if $f_{\lambda_{j}}(e(p)) \cdot \nu(e(p))=0$ for all $p \in V$ and $j=1, \ldots, r$.

Proof. Suppose that $e$ is a pre-envelope of $(f, \nu)$. We denote $f=\left(f_{1}, \ldots, f_{n+1}\right), \nu=$ $\left(\nu_{1}, \ldots, \nu_{n+1}\right)$. By a direct calculation,

$$
\begin{aligned}
E_{p_{i}}(p)= & \frac{\partial}{\partial p_{i}}(f \circ e(p)) \\
= & \left(\sum_{k=1}^{n} f_{1 u_{k}}(e(p)) u_{k p_{i}}(p)+\sum_{j=1}^{r} f_{1 \lambda_{j}}(e(p)) \lambda_{j p_{i}}(p), \ldots,\right. \\
& \left.\sum_{k=1}^{n} f_{n+1 u_{k}}(e(p)) u_{k p_{i}}(p)+\sum_{j=1}^{r} f_{n+1 \lambda_{j}}(e(p)) \lambda_{j p_{i}}(p)\right) .
\end{aligned}
$$

Since $E_{p_{i}}(p) \cdot \nu(e(p))=0$ for all $p \in V$ and $i=1, \ldots, n$, and $(f, \nu)$ is an $r$-parameter family of Legendre mappings, we have

$$
\left(f_{\lambda_{1}}(e(p)) \cdot \nu(e(p))\right) \lambda_{1 p_{i}}(p)+\cdots+\left(f_{\lambda_{r}}(e(p)) \cdot \nu(e(p))\right) \lambda_{r p_{i}}(p)=0
$$

for all $p \in V$ and $i=1, \ldots, n$. It follows that

$$
\left(\begin{array}{ccc}
\lambda_{1 p_{1}}(p) & \cdots & \lambda_{r p_{1}}(p) \\
\vdots & \cdots & \vdots \\
\lambda_{1 p_{n}}(p) & \cdots & \lambda_{r p_{n}}(p)
\end{array}\right)\left(\begin{array}{c}
f_{\lambda_{1}}(e(p)) \cdot \nu(e(p)) \\
\vdots \\
f_{\lambda_{r}}(e(p)) \cdot \nu(e(p))
\end{array}\right)=\left(\begin{array}{c}
0 \\
\vdots \\
0
\end{array}\right) .
$$

By the assumption $n \geq r$ and the variability condition, we have $f_{\lambda_{j}}(e(p)) \cdot \nu(e(p))=0$ for all $p \in V$ and $j=1, \ldots, r$. 
Conversely, suppose that $f_{\lambda_{j}}(e(p)) \cdot \nu(e(p))=0$ for all $p \in V$ and $j=1, \ldots, r$. By a direct calculation, we have

$$
\begin{aligned}
E_{p_{i}}(p) \cdot \nu(e(p))= & \left(\sum_{k=1}^{n} f_{1 u_{k}}(e(p)) u_{k p_{i}}(p)+\sum_{j=1}^{r} f_{1 \lambda_{j}}(e(p)) \lambda_{j p_{i}}(p)\right) \cdot \nu_{1}(e(p)) \\
& +\cdots+\left(\sum_{k=1}^{n} f_{n+1 u_{k}}(e(p)) u_{k p_{i}}(p)+\sum_{j=1}^{r} f_{n+1 \lambda_{j}}(e(p)) \lambda_{j p_{i}}(p)\right) \cdot \nu_{n+1}(e(p)) \\
= & \sum_{k=1}^{n} u_{k p_{i}}(p) f_{u_{k}}(e(p)) \cdot \nu(e(p))+\sum_{j=1}^{r} \lambda_{j p_{i}}(p) f_{\lambda_{j}}(e(p)) \cdot \nu(e(p)) \\
= & 0
\end{aligned}
$$

for all $p \in V$ and $i=1, \ldots, n$. It follows that $e$ is a pre-envelope of $(f, \nu)$.

Remark 3.7 In Theorem 3.6, the assumption $n \geq r$ does not need to prove the converse.

Example 3.8 Let $(f, \nu): \mathbb{R}^{2} \times \mathbb{R} \rightarrow \mathbb{R}^{3} \times S^{2}$ be

$$
f(u, v, \lambda)=\left(u^{2}+\lambda, u^{3}, v\right), \nu(u, v, \lambda)=\frac{1}{\sqrt{9 u^{2}+4}}(3 u,-2,0) .
$$

Then $(f, \nu)$ is a one-parameter family of Legendre mappings (immersions) and $f$ is the cuspidal edge for each fixed $\lambda \in \mathbb{R}$. Since $f_{\lambda}(u, v, \lambda) \cdot \nu(u, v, \lambda)=3 u / \sqrt{9 u^{2}+4}$, if we take $e: \mathbb{R}^{2} \rightarrow$ $\mathbb{R}^{2} \times \mathbb{R}, e(p, q)=(0, p, q)$, then the variability condition holds and $f_{\lambda}(e(p, q)) \cdot \nu(e(p, q))=0$ for all $(p, q) \in \mathbb{R}^{2}$. By Theorem 3.6, $e$ is a pre-envelope and $E(p, q)=f \circ e(p, q)=(q, 0, p)$ is an envelope. Hence $x z$-plane is an envelope of $(f, \nu)$, see Example 2.2.

Example 3.9 Let $(f, \nu): \mathbb{R}^{2} \times \mathbb{R} \rightarrow \mathbb{R}^{3} \times S^{2}$ be

$$
f(u, v, \lambda)=\left(u^{2}, u^{3}+\lambda, v\right), \nu(u, v, \lambda)=\frac{1}{\sqrt{9 u^{2}+4}}(3 u,-2,0) .
$$

Then $(f, \nu)$ is a one-parameter family of Legendre mappings (immersions) and $f$ is the cuspidal edge for each fixed $\lambda \in \mathbb{R}$. Since $f_{\lambda}(u, v, \lambda) \cdot \nu(u, v, \lambda)=-2 / \sqrt{9 u^{2}+4} \neq 0,(f, \nu)$ does not have an envelope by Theorem 3.6. Hence $y z$-plane is not an envelope of $(f, \nu)$, see Example 2.3.

Definition 3.10 We say that a map $\Phi: \widetilde{U} \times \widetilde{\Lambda} \rightarrow U \times \Lambda$ is an $r$-parameter family of parameter change if $\Phi$ is a diffeomorphism and given by the form $\Phi(q, k)=(\phi(q, k), \varphi(k))$.

Proposition 3.11 Let $(f, \nu): U \times \Lambda \rightarrow \mathbb{R}^{n+1} \times S^{n}$ be an r-parameter family of Legendre mappings. Suppose that $n \geq r, e: V \rightarrow U \times \Lambda$ is a pre-envelope, $E=f \circ e$ is an envelope and $\Phi: \widetilde{U} \times \widetilde{\Lambda} \rightarrow U \times \Lambda$ is an r-parameter family of parameter change. Then $(\widetilde{f}, \widetilde{\nu})=$ $(f \circ \Phi, \nu \circ \Phi): \widetilde{U} \times \widetilde{\Lambda} \rightarrow \mathbb{R}^{n+1} \times S^{n}$ is also an r-parameter family of Legendre mappings. Moreover, $\Phi^{-1}$ o $e: V \rightarrow \widetilde{U} \times \widetilde{\Lambda}$ is a pre-envelope and $E$ is also an envelope of $(\widetilde{f}, \widetilde{\nu})$.

Proof. By the chain rule, we have $d(f \circ \Phi) \cdot \nu \circ \Phi=d f(\Phi) d \Phi \cdot \nu(\Phi)=0$ for fixed $k \in \widetilde{\Lambda}$. Therefore, $(\widetilde{f}, \widetilde{\nu})$ is also an $r$-parameter family of Legendre mappings. Since the form of $\Phi$, 
there exists a smooth map $\psi: U \times \Lambda \rightarrow \widetilde{U}$ such that $\Phi^{-1}(u, \lambda)=\left(\psi(u, \lambda), \varphi^{-1}(\lambda)\right)$. It follows that $\Phi^{-1} \circ e$ satisfies the variability condition. By a direct calculation, we have

$$
\begin{aligned}
\widetilde{f}_{k_{j}}(q, k)= & \frac{\partial}{\partial k_{j}} f \circ \Phi(q, k) \\
= & \left(\sum_{i=1}^{n} f_{1 u_{i}}(\Phi(q, k)) \phi_{i k_{j}}(q, k)+\sum_{\ell=1}^{r} f_{1 \lambda_{\ell}}(\Phi(q, k)) \varphi_{\ell k_{j}}(k), \cdots\right. \\
& \left.\sum_{i=1}^{n} f_{n+1 u_{i}}(\Phi(q, k)) \phi_{i k_{j}}(q, k)+\sum_{\ell=1}^{r} f_{n+1 \lambda_{\ell}}(\Phi(q, k)) \varphi_{\ell k_{j}}(k)\right)
\end{aligned}
$$

for all $(q, k) \in \widetilde{U} \times \widetilde{\Lambda}$ and $j=1, \ldots, r$. Then

$\widetilde{f}_{k_{j}}\left(\Phi^{-1} \circ e(p)\right) \cdot \widetilde{\nu}\left(\Phi^{-1} \circ e(p)\right)=\varphi_{1 k_{j}}(\lambda(p)) f_{\lambda_{1}}(e(p)) \cdot \nu(e(p))+\cdots+\varphi_{r k_{j}}(\lambda(p)) f_{\lambda_{r}}(e(p)) \cdot \nu(e(p))=0$

for all $p \in V$ and $j=1, \ldots, r$. It follows that $\Phi^{-1} \circ e$ is a pre-envelope of $(\widetilde{f}, \widetilde{\nu})$, and hence $\widetilde{f} \circ \Phi^{-1} \circ e=f \circ \Phi \circ \Phi^{-1} \circ e=f \circ e=E$ is also an envelope of $(\widetilde{f}, \widetilde{\nu})$.

Let $(f, \nu): U \times \Lambda \rightarrow \mathbb{R}^{n+1} \times S^{n}$ be an $r$-parameter family of Legendre mappings. We define the parallel of the $r$-parameter family of Legendre mappings by $f^{k}: U \times \Lambda \rightarrow \mathbb{R}^{n+1}, f^{k}(u, \lambda)=$ $f(u, \lambda)+k \nu(u, \lambda)$, where $k \in \mathbb{R}$. It is easy to see that $\left(f^{k}, \nu\right)$ is also an $r$-parameter family of Legendre mappings for each fixed $k \in \mathbb{R}$.

Proposition 3.12 Suppose that $e: V \rightarrow U \times \Lambda$ is a pre-envelope of $(f, \nu)$ (and $E$ is an envelope) and $n \geq r$. Then the envelope of the parallel of the $r$-parameter family of Legendre mappings is given by the parallel of the envelope.

Proof. Since $\nu$ is a unit vector, $\nu_{\lambda_{j}}(u, \lambda) \cdot \nu(u, \lambda)=0$. Therefore, $f_{\lambda j}^{k}(u, \lambda) \cdot \nu(u, \lambda)=\left(f_{\lambda j}(u, \lambda)+\right.$ $\left.k \nu_{\lambda j}(u, \lambda)\right) \cdot \nu(u, \lambda)=f_{\lambda j}(u, \lambda) \cdot \nu(u, \lambda)$. If $e$ is a pre-envelope of $(f, \nu)$, then $f_{\lambda_{j}}^{k}(e(p)) \cdot \nu(e(p))=$ $f_{\lambda_{j}}(e(p)) \cdot \nu(e(p))=0$ for all $p \in V$ and $j=1, \ldots, r$. It follows that $e$ is also a pre-envelope of $\left(f^{k}, \nu\right)$ by Theorem 3.6. By definition, the envelope of the parallel of the $r$-parameter family of Legendre mappings is given by $E^{k}=f^{k} \circ e=f \circ e+k \nu \circ e=E+k \nu \circ e$. It follows that $E^{k}$ is the parallel of the Legendre mapping $(E, \nu \circ e)$.

We give a relation between the envelope $E_{I}$ of the classical definition by using an implicit function (Definition 2.1) and the envelope $E$ of an $r$-parameter family of Legendre mappings (Definition 3.1).

Proposition 3.13 Let $(f, \nu): U \times \Lambda \rightarrow \mathbb{R}^{n+1} \times S^{n}$ be an r-parameter family of Legendre mappings, and let $F(x, \lambda)=0$ be an implicit function of the r-parameter family of frontals, that is, assume $F(f(u, \lambda), \lambda)=0$ and $\left(F_{x_{1}}, \ldots, F_{x_{n+1}}\right)(f(u, \lambda), \lambda)$ is parallel to $\nu(u, \lambda)$ for all $(u, \lambda) \in U \times \Lambda$. Suppose that $n \geq r$. If $e: V \rightarrow U \times \Lambda$ is a pre-envelope and $E: V \rightarrow \mathbb{R}^{n+1}$ is an envelope of $(f, \nu)$, then $E(V) \subset E_{I}$.

Proof. By differentiating $F(f(u, \lambda), \lambda)=0$ with respect to $\lambda_{j}$, we have

$$
F_{x_{1}}(f(u, \lambda), \lambda) f_{1 \lambda_{j}}(u, \lambda)+\cdots+F_{x_{n+1}}(f(u, \lambda), \lambda) f_{n+1 \lambda_{j}}(u, \lambda)+F_{\lambda_{j}}(f(u, \lambda), \lambda)=0,
$$

where $j=1, \ldots, r$. By the assumption, there exists a smooth function $a: U \times \Lambda \rightarrow \mathbb{R}$ such that $\left(F_{x_{1}}, \ldots, F_{x_{n+1}}\right)(f(u, \lambda), \lambda)=a(u, \lambda)\left(\nu_{1}, \ldots, \nu_{n+1}\right)(u, \lambda)$ for all $(u, \lambda) \in U \times \Lambda$. By 
Theorem 3.6, we have $f_{\lambda_{j}}(e(p)) \cdot \nu(e(p))=0$ for all $p \in V$ and $j=1, \ldots, r$. It follows that $F_{\lambda_{j}}(f(e(p)), \lambda(p))=0$ for all $p \in V$ and $j=1, \ldots, r$. Therefore $E(p) \in E_{I}$ with respect to $\lambda(p)$ for all $p \in V$.

Proposition 3.14 Let $(f, \nu): U \times \Lambda \rightarrow \mathbb{R}^{n+1} \times S^{n}$ be an r-parameter family of Legendre mappings, and let $e: V \rightarrow U \times \Lambda$ be a smooth map satisfying the variability condition. If $\operatorname{rank}\left(f_{u_{1}}, \ldots, f_{u_{n}}\right)(e(p))=n$ and the trace of $e$ lies in the singular set of $f$, then $e$ is a preenvelope of $(f, \nu)$ (and $E$ is an envelope).

Proof. We denote the set of singular points (singular set) of $f$ by $\Sigma(f)$. Since $e(p) \in \Sigma(f)$, we have the condition

$$
\operatorname{rank}\left(\begin{array}{cccccc}
f_{1 u_{1}} & \cdots & f_{1 u_{n}} & f_{1 \lambda_{1}} & \cdots & f_{1 \lambda_{r}} \\
\vdots & & \vdots & \vdots & & \vdots \\
f_{n+1 u_{1}} & \cdots & f_{n+1 u_{n}} & f_{n+1 \lambda_{1}} & \cdots & f_{n+1 \lambda_{r}}
\end{array}\right)(e(p))<n+1 .
$$

By the assumption $\operatorname{rank}\left(f_{u_{1}}, \ldots, f_{u_{n}}\right)(e(p))=n$, there exist smooth functions $a_{i j}: V \rightarrow \mathbb{R}$, $i=1, \ldots, n, j=1 \ldots, r$ such that $f_{\lambda_{j}}(e(p))=a_{1 j}(p) f_{u_{1}}(e(p))+\cdots+a_{n j}(p) f_{u_{n}}(e(p))$. It follows that $f_{\lambda_{j}}(e(p)) \cdot \nu(e(p))=0$ for all $p \in V$ and $j=1, \ldots, r$. Hence $e$ is a pre-envelope of $(f, \nu)$.

Proposition 3.15 Let $(f, \nu): U \times \Lambda \rightarrow \mathbb{R}^{n+1} \times S^{n}$ be an r-parameter family of Legendre mappings, and let $F(x, \lambda)=0$ be an implicit function of the r-parameter family of frontals, that is, assume $F(f(x, \lambda), \lambda)=0$ and $\left(F_{x_{1}}, \ldots, F_{x_{n+1}}\right)(f(u, \lambda), \lambda)$ is parallel to $\nu(u, \lambda)$ for all $(u, \lambda) \in U \times \Lambda$. Suppose that $e: V \rightarrow U \times \Lambda, e(p)=(u(p), \lambda(p))$ is a smooth mapping satisfying the variability condition. If $E(p)=f \circ e(p) \in E_{I}$ with respect to $\lambda(p), \operatorname{rank}\left(f_{u_{1}}, \ldots, f_{u_{n}}\right)(e(p))=$ $n$ and

$$
\left(F_{x_{1}}, \ldots, F_{x_{n+1}}\right)(f(e(p)), \lambda(p)) \neq(0, \ldots, 0)
$$

for all $p \in V$, then $e$ is a pre-envelope of $(f, \nu)$ (and $E$ is an envelope).

Proof. By differentiating $F(f(u, \lambda), \lambda)=0$ with respect to $u_{i}$ and $\lambda_{j}$, we have

$$
\begin{aligned}
& F_{x_{1}}(f(u, \lambda), \lambda) f_{1 u_{i}}(u, \lambda)+\cdots+F_{x_{n+1}}(f(u, \lambda), \lambda) f_{n+1 u_{i}}(u, \lambda)=0, \\
& F_{x_{1}}(f(u, \lambda), \lambda) f_{1 \lambda_{j}}(u, \lambda)+\cdots+F_{x_{n+1}}(f(u, \lambda), \lambda) f_{n+1 \lambda_{j}}(u, \lambda)+F_{\lambda_{j}}(f(u, \lambda), \lambda)=0,
\end{aligned}
$$

where $i=1, \ldots, n, j=1, \ldots, r$. Since $E(p) \in E_{I}$ with respect to $\lambda(p)$, we have $F_{\lambda_{j}}(f(e(p)), \lambda(p))=$ 0 for all $p \in V$ and $j=1, \ldots, r$. It follows that

$$
\left(\begin{array}{ccc}
f_{1 u_{1}}(e(p)) & \cdots & f_{n+1 u_{1}}(e(p)) \\
\vdots & & \vdots \\
f_{1 u_{n}}(e(p)) & \cdots & f_{n+1 u_{n}}(e(p)) \\
f_{1 \lambda_{1}}(e(p)) & \cdots & f_{n+1 \lambda_{1}}(e(p)) \\
\vdots & & \vdots \\
f_{1 \lambda_{r}}(e(p)) & \cdots & f_{n+1 \lambda_{r}}(e(p))
\end{array}\right)\left(\begin{array}{c}
F_{x_{1}}(f(e(p)), \lambda(p)) \\
\vdots \\
F_{x_{n+1}}(f(e(p)), \lambda(p))
\end{array}\right)=\left(\begin{array}{c}
0 \\
\vdots \\
0
\end{array}\right)
$$

If the $\operatorname{rank} d f(e(p))=n+1$, then $\left(F_{x_{1}}, \ldots, F_{x_{n+1}}\right)(f(e(p)), \lambda(p))=(0, \ldots, 0)$. By the assumption $\left(F_{x_{1}}, \ldots, F_{x_{n+1}}\right)(f(e(p)), \lambda(p)) \neq(0, \ldots, 0)$, we have rank $d f(e(p))<n+1$. It follows that $e(p) \in \Sigma(f)$. By Proposition 3.14, $e$ is a pre-envelope of $(f, \nu)$. 


\section{Singular solutions of first order partial differential equa- tions}

As an application of the theory of envelopes of families of Legendre mappings, we give a condition that the projection of a singular solution of a first order partial differential equation is an envelope.

We quickly review the theory of singular solutions and of Clairaut type of first order partial differential equations, in detail see $[15,16]$.

An equation is a submersion germ $F:\left(J^{1}\left(\mathbb{R}^{n}, \mathbb{R}\right), z_{0}\right) \rightarrow(\mathbb{R}, 0)$ on the 1 -jet space of functions of $n$-variables, where $z_{0}=\left(x_{0}, y_{0}, p_{0}\right)$. Let $\theta$ be a canonical contact 1 -form on $J^{1}\left(\mathbb{R}^{n}, \mathbb{R}\right)$ which is given by $\theta=d y-\sum_{i=1}^{n} p_{i} d x_{i}$, where $(x, y, p)=\left(x_{1}, \ldots, x_{n}, y, p_{1}, \ldots, p_{n}\right)$ is the canonical coordinate on $J^{1}\left(\mathbb{R}^{n}, \mathbb{R}\right)$. We define a geometric solution of $F=0$ to be an immersion germ $i:\left(L, q_{0}\right) \rightarrow\left(F^{-1}(0), z_{0}\right)$ of an $n$-dimensional manifold such that $i^{*} \theta=0$, that is, a Legendre submanifold which is contained in $F^{-1}(0)$. We say that $z_{0}$ is a contact singular point if $\theta\left(T_{z_{0}} F^{-1}(0)\right)=0$. It is easy to see that $z_{0}$ is a contact singular point if and only if $F=F_{p_{i}}=F_{x_{i}}+p_{i} F_{y}=0$ for $i=1, \ldots, n$ at $z_{0}$. We also say that $z_{0}$ is a $\pi$-singular point if $F=F_{p_{i}}=0$ for $i=1, \ldots, n$ at $z_{0}$. We denote the set of contact singular points by $\Sigma_{c}(F)$, the set of $\pi$-singular points by $\Sigma_{\pi}(F)$ and $\pi\left(\Sigma_{\pi}(F)\right)=D_{F}$, where $\pi: J^{n}\left(\mathbb{R}^{n}, \mathbb{R}\right) \rightarrow \mathbb{R}^{n+1}$ is the canonical projection $\pi(x, y, p)=(x, y)$. We call the set $D_{F}$ a discriminant set of the equation $F=0$.

An equation $F=0$ is said to be completely integrable at $z_{0}$ if there exists a foliation by geometric solution on $F^{-1}(0)$ around $z_{0}$, that is, there exists an immersion germ $\Gamma:\left(\mathbb{R}^{n} \times\right.$ $\left.\mathbb{R}^{n},\left(u_{0}, c_{0}\right)\right) \rightarrow\left(F^{-1}(0), z_{0}\right)$ such that $\Gamma(\cdot, c)$ is a geometric solution of $F=0$ for each $c \in$ $\left(\mathbb{R}^{n}, c_{0}\right)$. In this case, such a foliation is called a complete solution of $F=0$ at $z_{0}$. We say that an $n$-parameter family of function germs $f:\left(\mathbb{R}^{n} \times \mathbb{R}^{n},\left(x_{0}, c_{0}\right)\right) \rightarrow\left(\mathbb{R}, y_{0}\right)$ is a classical complete solution of $F=0$ at $z_{0}$ if a complete solution is a form of $j^{1} f:\left(\mathbb{R}^{n} \times \mathbb{R}^{n},\left(x_{0}, c_{0}\right)\right) \rightarrow\left(F^{-1}(0), z_{0}\right)$, that is, $F\left(x, f(x, c), f_{x}(x, c)\right)=0$ and $j^{1} f(x, c)=\left(x, f(x, c), f_{x}(x, c)\right)$ is an immersion germ. An equation $F=0$ is said to be classical completely integrable at $z_{0}$ if there exists a classical complete solution of $F=0$ at $z_{0}$.

A geometric solution $i:\left(L, q_{0}\right) \rightarrow\left(F^{-1}(0), z_{0}\right)$ of $F=0$ is called a singular solution of $F=0$ at $z_{0}$ if for any representative $\widetilde{i}: U \rightarrow F^{-1}(0)$ of $i$ and any open subset $V \subset U, \widetilde{i}(V)$ is not contained in a leaf of any complete solutions of $F=0$.

An equation $F=0$ is called of Clairaut type at $z_{0}$ if there exist smooth function germs $B_{j i}, A_{i}:\left(J^{1}\left(\mathbb{R}^{n}, \mathbb{R}\right), z_{0}\right) \rightarrow \mathbb{R}$ for $i, j=1, \ldots, n$ such that

$$
F_{x_{i}}+p_{i} F_{y}=\sum_{j=1}^{n} B_{j i} F_{p_{j}}+A_{i} F, B_{j i}=B_{i j}
$$

and

$$
\frac{\partial B_{j k}}{\partial x_{i}}+p_{i} \frac{\partial B_{j k}}{\partial y}+\sum_{\ell=1}^{n} B_{\ell i} \frac{\partial B_{j k}}{\partial p_{\ell}}=\frac{\partial B_{j i}}{\partial x_{k}}+p_{k} \frac{\partial B_{j i}}{\partial y}+\sum_{\ell=1}^{n} B_{\ell k} \frac{\partial B_{j i}}{\partial p_{\ell}}
$$

at any $(x, y, p) \in\left(F^{-1}(0), z_{0}\right)$ for $i, j, k=1, \ldots, n$. Then we have the following result.

Theorem $4.1([\mathbf{1 5}, \mathbf{1 6}])$ Let $F:\left(J^{1}\left(\mathbb{R}^{n}, \mathbb{R}\right), z_{0}\right) \rightarrow(\mathbb{R}, 0)$ be a first order partial differential equation germs. 
(1) $F=0$ is completely integrable at $z_{0}$ if and only if $\Sigma_{c}(F)=\emptyset$ or $\Sigma_{c}(F)$ is an $n$ dimensional submanifold around $z_{0}$. Moreover, if $\Sigma_{c}(F) \neq \emptyset$, then $\Sigma_{c}(F)$ is a singular solution of $F=0$ at $z_{0}$.

(2) $F=0$ is smooth completely integrable at $z_{0}$ if and only if $F=0$ is of Clairaut type at $z_{0}$. In this case, if $\Sigma_{\pi}(F) \neq \emptyset$, then $\Sigma_{\pi}(F)$ is a singular solution of $F=0$ at $z_{0}$ and the discriminant set $D_{F}$ is the envelope of the family of graphs of the smooth complete solution.

By using the envelope theorem (Theorem 3.6), we have the following result.

Theorem 4.2 Let $F:\left(J^{1}\left(\mathbb{R}^{n}, \mathbb{R}\right), z_{0}\right) \rightarrow(\mathbb{R}, 0)$ be a first order partial differential equation germs and not of Clairaut type at $z_{0}$. Suppose that $\Gamma=(x, y, p):\left(\mathbb{R}^{n} \times \mathbb{R}^{n},\left(u_{0}, c_{0}\right)\right) \rightarrow$ $\left(F^{-1}(0), z_{0}\right)$ is a complete solution of $F=0$ at $z_{0}, \Sigma_{c}(F)=\Sigma_{\pi}(F) \neq \emptyset$ and $e:\left(\mathbb{R}^{n}, \widetilde{q}_{0}\right) \rightarrow$ $\left(\mathbb{R}^{n} \times \mathbb{R}^{n},\left(u_{0}, c_{0}\right)\right)$ is a smooth mapping satisfying the variability condition. Then $e$ is a preenvelope and $E=\pi \circ \Gamma \circ e$ is an envelope of $(\pi \circ \Gamma, \nu)$ if and only if $E(q) \in \pi\left(\Sigma_{c}(F)\right)$ for all $q \in\left(\mathbb{R}^{n}, \widetilde{q}_{0}\right)$, where $\nu(u, c)=(-p(u, c), 1) / \sqrt{1+|p(u, c)|^{2}}$.

Proof. By the assumption and Theorem $4.1(1), \Sigma_{c}(F)=\Sigma_{\pi}(F)$ is an $n$-dimensional manifold around $z_{0}$ and a singular solution of $F=0$ at $z_{0}$. Since $z_{0} \in \Sigma_{c}(F)$ and $F=0$ is submersion, we may consider $F(x, y, p)=-y+g(x, p)$, where $g$ is a smooth function, $x=\left(x_{1}, \ldots, x_{n}\right)$ and $p=\left(p_{1}, \ldots, p_{n}\right)$. We denote the complete solution of $F=0$ at $z_{0}$ by

$$
\Gamma(u, c)=(x(u, c), y(u, c), p(u, c))=\left(x_{1}(u, c), \ldots, x_{n}(u, c), y(u, c), p_{1}(u, c), \ldots, p_{n}(u, c)\right),
$$

where $u=\left(u_{1}, \ldots, u_{n}\right), c=\left(c_{1}, \ldots, c_{n}\right)$. Since $y(u, c)=g(x(u, c), p(u, c))$ and $\Gamma^{*} \theta=0$, we have $y_{u_{i}}(u, c)=p_{1}(u, c) x_{1 u_{i}}(u, c)+\cdots+p_{n}(u, c) x_{n u_{i}}(u, c)$ for $i=1, \ldots, n$. Since $F=0$ is not of Clairaut type at $z_{0}$, we have

$$
\operatorname{rank}\left(\begin{array}{ccc}
x_{1 u_{1}} & \cdots & x_{n u_{1}} \\
\vdots & & \vdots \\
x_{1 u_{n}} & \cdots & x_{n u_{n}}
\end{array}\right)\left(u_{0}, c_{0}\right)<n
$$

and

$$
\begin{aligned}
& \operatorname{rank}\left(\begin{array}{ccccccc}
x_{1 u_{1}} & \cdots & x_{n u_{1}} & y_{u_{1}} & p_{1 u_{1}} & \cdots & p_{n u_{1}} \\
\vdots & & \vdots & \vdots & \vdots & & \vdots \\
x_{1 u_{n}} & \cdots & x_{n u_{n}} & y_{u_{n}} & p_{1 u_{n}} & \cdots & p_{n u_{n}}
\end{array}\right)\left(u_{0}, c_{0}\right) \\
& =\operatorname{rank}\left(\begin{array}{cccccc}
x_{1 u_{1}} & \cdots & x_{n u_{1}} & p_{1 u_{1}} & \cdots & p_{n u_{1}} \\
\vdots & & \vdots & \vdots & & \vdots \\
x_{1 u_{n}} & \cdots & x_{n u_{n}} & p_{1 u_{n}} & \cdots & p_{n u_{n}}
\end{array}\right)\left(u_{0}, c_{0}\right)=n .
\end{aligned}
$$

Set $f(u, c)=\pi \circ \Gamma(u, c)$ and $\nu(u, c)=(-p(u, c), 1) / \sqrt{1+|p(u, c)|^{2}}$. By a direct calculation, we have $f_{u_{i}}(u, c) \cdot \nu(u, c)=0$ for all $(u, c) \in\left(\mathbb{R}^{n} \times \mathbb{R}^{n},\left(u_{0}, c_{0}\right)\right)$ and $i=1, \ldots, n$. It follows that $(f, \nu)$ is an $n$-parameter family of Legendre mappings (immersions). Moreover, we have

$$
\begin{aligned}
f_{c_{i}}(u, c)= & \left(x_{1 c_{i}}(u, c), \cdots, x_{n c_{i}}(u, c), y_{c_{i}}(u, c)\right) \\
= & \left(x_{1 c_{i}}(u, c), \cdots, x_{n c_{i}}(u, c),\right. \\
& \left.\quad \sum_{j=1}^{n} x_{j c_{i}}(u, c) g_{x_{j}}(x(u, c), p(u, c))+\sum_{j=1}^{n} p_{j c_{i}}(u, c) g_{p_{j}}(x(u, c), p(u, c))\right) .
\end{aligned}
$$


It follows that $f_{c_{i}}(u, c) \cdot \nu(u, c)=$

$$
\frac{1}{\sqrt{1+|p(u, c)|^{2}}}\left(\sum_{j=1}^{n}\left(-p_{j}(u, c)+g_{x_{j}}(x(u, c), p(u, c))\right) x_{j c_{i}}(u, c)+\sum_{j=1}^{n} p_{j c_{i}}(u, c) g_{p_{j}}(x(u, c), p(u, c))\right) .
$$

We now consider the following case. Suppose that

$$
\operatorname{rank}\left(\begin{array}{ccc}
p_{1 u_{1}} & \cdots & p_{n u_{1}} \\
\vdots & & \vdots \\
p_{1 u_{n}} & \cdots & p_{n u_{n}}
\end{array}\right)\left(u_{0}, c_{0}\right)=n
$$

By using an $n$-parameter family of parameter change, we may assume that $p_{i}(u, c)=u_{i}$ for $i=1, \ldots, n$ by Proposition 3.11. If $e:\left(\mathbb{R}^{n}, \widetilde{q_{0}}\right) \rightarrow\left(\mathbb{R}^{n} \times \mathbb{R}^{n},\left(u_{0}, c_{0}\right)\right)$ is a pre-envelope of $(f, \nu)$, then

$$
\left(\begin{array}{ccc}
x_{1 c_{1}}(e(q)) & \cdots & x_{1 c_{n}}(e(q)) \\
\vdots & & \vdots \\
x_{n c_{n}}(e(q)) & \cdots & x_{n c_{n}}(e(q))
\end{array}\right)\left(\begin{array}{c}
\left(-p_{1}+g_{x_{1}}\right)(e(q)) \\
\vdots \\
\left(-p_{n}+g_{x_{n}}\right)(e(q))
\end{array}\right)=\left(\begin{array}{c}
0 \\
\vdots \\
0
\end{array}\right) .
$$

Here we denote a local coordinate $\left(\mathbb{R}^{n}, \widetilde{q_{0}}\right)$ by $q$ instead of $p$ in $\S 3$. Since $\Gamma$ is an immersion germ and by Theorem 3.6, we have $\left(-p_{i}+g_{x_{i}}\right)(e(q))=0$ for $i=1, \ldots, n$. It follows that $g_{p_{i}}(e(q))=0$ for $i=1, \ldots, n$ and hence $E(q) \in \pi\left(\Sigma_{\pi}(F)\right)=\pi\left(\Sigma_{c}(F)\right)$. Conversely, if $E(q) \in \pi\left(\Sigma_{c}(F)\right)$, then $f_{c_{i}}(e(q)) \cdot \nu(e(q))=0$ for all $i=1, \ldots, n$. By Theorem 3.6, $e$ is a pre-envelope of $(f, \nu)$.

Moreover, suppose that

$$
\operatorname{rank}\left(\begin{array}{cccccc}
x_{1 u_{1}} & \cdots & x_{n u_{k}} & p_{k+1 u_{1}} & \cdots & p_{n u_{1}} \\
\vdots & & \vdots & \vdots & & \vdots \\
x_{1 u_{n}} & \cdots & x_{n u_{k}} & p_{k+1 u_{n}} & \cdots & p_{n u_{n}}
\end{array}\right)\left(u_{0}, c_{0}\right)=n \text {. }
$$

By using an $n$-parameter family of parameter change, we may assume that $x_{i}(u, c)=u_{i}$ for $i=1, \ldots, k$ and $p_{j}(u, c)=u_{j}$ for $j=k+1, \ldots, n$ by Proposition 3.11. Then we also have $\left(-p_{i}+g_{x_{i}}\right)(e(q))=0$ for $i=1, \ldots, k$ and $g_{p_{j}}(e(q))=0$ for $j=k+1, \ldots, n$. It follows that $g_{p_{i}}(e(q))=0$ for $i=1, \ldots, k$ and hence $E(q) \in \pi\left(\Sigma_{\pi}(F)\right)=\pi\left(\Sigma_{c}(F)\right)$. Conversely, if $E(q) \in \pi\left(\Sigma_{c}(F)\right)$, then $f_{c_{i}}(e(q)) \cdot \nu(e(q))=0$ for all $i=1, \ldots, n$. By Theorem 3.6, $e$ is a pre-envelope of $(f, \nu)$.

The other cases, we can also prove by similarly. This completes the proof of Theorem.

By Theorems 4.1 and 4.2, if $\Sigma_{c}(F)=\Sigma_{\pi}(F)$ is an $n$-dimensional submanifold around $z_{0}$, then $\Sigma_{c}(F)$ is a singular solution of $F=0$ at $z_{0}$ and the projection $\pi\left(\Sigma_{c}(F)\right)$ is an envelope when the variability condition holds.

We give concrete examples for completely integrable partial differential equations with a singular solution.

Example 4.3 Let $F: J^{1}\left(\mathbb{R}^{2}, \mathbb{R}\right) \rightarrow \mathbb{R}$ be given by $F\left(x_{1}, x_{2}, y, p_{1}, p_{2}\right)=-y+p_{1}^{n_{1}}+p_{2}^{n_{2}}=0$, where $n_{1}, n_{2} \geq 2$. That is, we consider the partial differential equation

$$
y=\left(\frac{\partial y}{\partial x_{1}}\right)^{n_{1}}+\left(\frac{\partial y}{\partial x_{2}}\right)^{n_{2}} .
$$


Then $\Sigma_{c}(F)=\Sigma_{\pi}(F)=\left\{\left(x_{1}, x_{2}, 0,0,0\right)\right\}$ is a 2-dimensional submanifold. The complete solution $\Gamma: \mathbb{R}^{2} \times \mathbb{R}^{2} \rightarrow F^{-1}(0)$ is given by

$$
\Gamma\left(u_{1}, u_{2}, c_{1}, c_{2}\right)=\left(\frac{n_{1}}{n_{1}-1} u_{1}^{n_{1}-1}+c_{1}, \frac{n_{2}}{n_{2}-1} u_{2}^{n_{2}-1}+c_{2}, u_{1}^{n_{1}}+u_{2}^{n_{2}}, u_{1}, u_{2}\right) .
$$

Then $(f, \nu): \mathbb{R}^{2} \times \mathbb{R}^{2} \rightarrow \mathbb{R}^{3} \times S^{2}$ is a 2-parameter family of Legendre mappings, where

$$
\begin{aligned}
& f\left(u_{1}, u_{2}, c_{1}, c_{2}\right)=\pi \circ \Gamma\left(u_{1}, u_{2}, c_{1}, c_{2}\right)=\left(\frac{n_{1}}{n_{1}-1} u_{1}^{n_{1}-1}+c_{1}, \frac{n_{2}}{n_{2}-1} u_{2}^{n_{2}-1}+c_{2}, u_{1}^{n_{1}}+u_{2}^{n_{2}}\right), \\
& \nu\left(u_{1}, u_{2}, c_{1}, c_{2}\right)=\frac{1}{\sqrt{1+u_{1}^{2}+u_{2}^{2}}}\left(-u_{1},-u_{2}, 1\right) .
\end{aligned}
$$

Since

$$
f_{c_{i}}\left(u_{1}, u_{2}, c_{1}, c_{2}\right) \cdot \nu\left(u_{1}, u_{2}, c_{1}, c_{2}\right)=-u_{i} / \sqrt{1+u_{1}^{2}+u_{2}^{2}}, i=1,2,
$$

$e: \mathbb{R}^{2} \rightarrow \mathbb{R}^{2} \times \mathbb{R}^{2}, e\left(q_{1}, q_{2}\right)=\left(0,0, q_{1}, q_{2}\right)$ is a pre-envelope and hence $E(q)=f \circ e(q)=$ $\left(q_{1}, q_{2}, 0\right) \in \pi\left(\Sigma_{c}(F)\right)$ is an envelope of $(f, \nu)$.

Example 4.4 Let $F: J^{1}\left(\mathbb{R}^{2}, \mathbb{R}\right) \rightarrow \mathbb{R}$ be given by $F\left(x_{1}, x_{2}, y, p_{1}, p_{2}\right)=-y+p_{1}^{n_{1}}+x_{2} p_{2}+x_{2}^{n_{2}}=0$, where $n_{1}, n_{2} \geq 2$. That is, we consider the partial differential equation

$$
y=\left(\frac{\partial y}{\partial x_{1}}\right)^{n_{1}}+x_{2} \frac{\partial y}{\partial x_{2}}+x_{2}^{n_{2}}
$$

Then $\Sigma_{c}(F)=\Sigma_{\pi}(F)=\left\{\left(x_{1}, 0,0,0, p_{2}\right)\right\}$ is a 2-dimensional submanifold. The complete solution $\Gamma: \mathbb{R}^{2} \times \mathbb{R}^{2} \rightarrow F^{-1}(0)$ is given by

$$
\Gamma\left(u_{1}, u_{2}, c_{1}, c_{2}\right)=\left(\frac{n_{1}}{n_{1}-1} u_{1}^{n_{1}-1}+c_{1}, u_{2}, u_{1}^{n_{1}}+\frac{2 n_{2}-1}{n_{2}-1} u_{2}^{n_{2}}+c_{2} u_{2}, u_{1}, \frac{n_{2}}{n_{2}-1} u_{2}^{n_{2}-1}+c_{2}\right) .
$$

Then $(f, \nu): \mathbb{R}^{2} \times \mathbb{R}^{2} \rightarrow \mathbb{R}^{3} \times S^{2}$ is a 2-parameter family of Legendre mappings, where

$$
\begin{aligned}
& f\left(u_{1}, u_{2}, c_{1}, c_{2}\right)=\pi \circ \Gamma\left(u_{1}, u_{2}, c_{1}, c_{2}\right)=\left(\frac{n_{1}}{n_{1}-1} u_{1}^{n_{1}-1}+c_{1}, u_{2}, u_{1}^{n_{1}}+\frac{2 n_{2}-1}{n_{2}-1} u_{2}^{n_{2}}+c_{2} u_{2}\right), \\
& \nu\left(u_{1}, u_{2}, c_{1}, c_{2}\right)=\frac{1}{\sqrt{1+u_{1}^{2}+\left(\frac{n_{2}}{n_{2}-1} u_{2}^{n_{2}-1}+c_{2}\right)^{2}}}\left(-u_{1},-\frac{n_{2}}{n_{2}-1} u_{2}^{n_{2}-1}-c_{2}, 1\right) .
\end{aligned}
$$

Since

$$
\begin{aligned}
& f_{c_{1}}\left(u_{1}, u_{2}, c_{1}, c_{2}\right) \cdot \nu\left(u_{1}, u_{2}, c_{1}, c_{2}\right)=-\frac{u_{1}}{\sqrt{1+u_{1}^{2}+\left(\frac{n_{2}}{n_{2}-1} u_{2}^{n_{2}-1}+c_{2}\right)^{2}}}, \\
& f_{c_{2}}\left(u_{1}, u_{2}, c_{1}, c_{2}\right) \cdot \nu\left(u_{1}, u_{2}, c_{1}, c_{2}\right)=\frac{u_{2}}{\sqrt{1+u_{1}^{2}+\left(\frac{n_{2}}{n_{2}-1} u_{2}^{n_{2}-1}+c_{2}\right)^{2}}}
\end{aligned}
$$

$e: \mathbb{R}^{2} \rightarrow \mathbb{R}^{2} \times \mathbb{R}^{2}, e\left(q_{1}, q_{2}\right)=\left(0,0, q_{1}, q_{2}\right)$ is a pre-envelope and hence $E(q)=f \circ e(q)=$ $\left(q_{1}, 0,0\right) \in \pi\left(\Sigma_{c}(F)\right)$ is an envelope of $(f, \nu)$. 
Example 4.5 Let $F: J^{1}\left(\mathbb{R}^{2}, \mathbb{R}\right) \rightarrow \mathbb{R}$ be given by $F\left(x_{1}, x_{2}, y, p_{1}, p_{2}\right)=-y+p_{1}^{n_{1}}+x_{2} p_{2}+g\left(p_{2}\right)=$ 0 , where $n_{1} \geq 2$ and $g$ is a smooth function. That is, we consider the partial differential equation

$$
y=\left(\frac{\partial y}{\partial x_{1}}\right)^{n_{1}}+x_{2} \frac{\partial y}{\partial x_{2}}+g\left(\frac{\partial y}{\partial x_{2}}\right) .
$$

Then $\Sigma_{c}(F)=\Sigma_{\pi}(F)=\left\{\left(x_{1},-g^{\prime}\left(p_{2}\right),-g^{\prime}\left(p_{2}\right) p_{2}+g\left(p_{2}\right), 0, p_{2}\right)\right\}$ is a 2-dimensional submanifold. The complete solution $\Gamma: \mathbb{R}^{2} \times \mathbb{R}^{2} \rightarrow F^{-1}(0)$ is given by

$$
\Gamma\left(u_{1}, u_{2}, c_{1}, c_{2}\right)=\left(\frac{n_{1}}{n_{1}-1} u_{1}^{n_{1}-1}+c_{1}, u_{2}, u_{1}^{n_{1}}+c_{2} u_{2}+g\left(c_{2}\right), u_{1}, c_{2}\right) .
$$

Then $(f, \nu): \mathbb{R}^{2} \times \mathbb{R}^{2} \rightarrow \mathbb{R}^{3} \times S^{2}$ is a 2-parameter family of Legendre mappings, where

$$
\begin{aligned}
& f\left(u_{1}, u_{2}, c_{1}, c_{2}\right)=\pi \circ \Gamma\left(u_{1}, u_{2}, c_{1}, c_{2}\right)=\left(\frac{n_{1}}{n_{1}-1} u_{1}^{n_{1}-1}+c_{1}, u_{2}, u_{1}^{n_{1}}+c_{2} u_{2}+g\left(c_{2}\right)\right), \\
& \nu\left(u_{1}, u_{2}, c_{1}, c_{2}\right)=\frac{1}{\sqrt{1+u_{1}^{2}+c_{2}^{2}}}\left(-u_{1},-c_{2}, 1\right) .
\end{aligned}
$$

Since

$$
\begin{aligned}
& f_{c_{1}}\left(u_{1}, u_{2}, c_{1}, c_{2}\right) \cdot \nu\left(u_{1}, u_{2}, c_{1}, c_{2}\right)=-u_{1} / \sqrt{1+u_{1}^{2}+u_{2}^{2}}, \\
& f_{c_{2}}\left(u_{1}, u_{2}, c_{1}, c_{2}\right) \cdot \nu\left(u_{1}, u_{2}, c_{1}, c_{2}\right)=\left(u_{2}+g^{\prime}\left(c_{2}\right)\right) / \sqrt{1+u_{1}^{2}+u_{2}^{2}},
\end{aligned}
$$

$e: \mathbb{R}^{2} \rightarrow \mathbb{R}^{2} \times \mathbb{R}^{2}, e\left(q_{1}, q_{2}\right)=\left(0,-g^{\prime}\left(q_{2}\right), q_{1}, q_{2}\right)$ is a pre-envelope and hence $E(q)=f \circ e(q)=$ $\left(q_{1},-g^{\prime}\left(q_{2}\right),-g^{\prime}\left(q_{2}\right) q_{2}+g\left(q_{2}\right)\right) \in \pi\left(\Sigma_{c}(F)\right)$ is an envelope of $(f, \nu)$.

\section{References}

[1] V. I. Arnol'd, Singularities of Caustics and Wave Fronts. Mathematics and Its Applications 62 Kluwer Academic Publishers (1990).

[2] V. I. Arnol'd, S. M. Gusein-Zade, A. N. Varchenko, Singularities of Differentiable Maps vol. I. Birkhäuser (1986).

[3] J. W. Bruce, P. J. Giblin, What is an envelope? Math. Gaz. 65 (1981), 186-192.

[4] J. W. Bruce, P. J. Giblin, Curves and Singularities. A geometrical introduction to singularity theory. Second edition. Cambridge University Press, Cambridge, 1992.

[5] J. W. Bruce, P. J. Giblin, C. G. Gibson, Caustics through the looking glass. Math. Intelligencer 6 (1984), 47-58.

[6] L. Chen, D. Pei, M. Takahashi, Envelopes of one-parameter families of frontals in hyperbolic and de-Sitter 2-space. Preprint. (2018).

[7] S. Ei, K. Fujii, T. Kunihiro, Renormalization-group method for reduction of evolution equations; invariant manifolds and envelopes. Ann. Physics. 280 (2000), 236-298.

[8] T. Fukunaga, M. Takahashi, Evolutes of fronts in the Euclidean plane. J. Singul. 10 (2014), 92-107. 
[9] T. Fukunaga, M. Takahashi, Evolutes and involutes of frontals in the Euclidean plane. Demonstr. Math. 48 (2015), 147-166.

[10] T. Fukunaga, M. Takahashi, Framed surfaces in the Euclidean space. Bull. Braz. Math. Soc. (N.S.) (2018). DOI:10.1007/s00574-018-0090-z.

[11] C. G. Gibson, Elementary Geometry of Differentiable Curves. An undergraduate introduction. Cambridge University Press, Cambridge, 2001.

[12] A. Gray, E. Abbena, S. Salamon, Modern differential geometry of curves and surfaces with Mathematica. Third edition. Studies in Advanced Mathematics. Chapman and Hall/CRC, Boca Raton, FL, 2006

[13] G. Ishikawa, Singularities of Curves and Surfaces in Various Geometric Problems. CAS Lecture Notes 10, Exact Sciences. 2015.

[14] G. Ishikawa, Singularities of frontals. Advanced Studies in Pure Mathematics. 78 (2018), 55-106.

[15] S. Izumiya, Singular solutions of first-order differential equations, Bull. London Math. Soc. 26 (1994), 69-74.

[16] S. Izumiya, On Clairaut-type equations, Publ. Math. Debrecen 45 (1995), 159-166.

[17] S. Izumiya, M. C. Romero-Fuster, M. A. S. Ruas, F. Tari, Differential Geometry from a Singularity Theory Viewpoint. World Scientific Pub. Co Inc. 2015.

[18] T. Kunihiro, A geometrical formulation of the renormalization group method for global analysis. Progr. Theoret. Phys. 94 (1995), 503-514.

[19] Y. Li, D. Pei, M. Takahashi, H. Yu, Envelopes of Legendre curves in the unit spherical bundle over the unit sphere. Quarterly J. Math. (2018) DOI: 10.1093/qmath/hax056.

[20] L. Martins, K. Saji, Geometric invariants of cuspidal edges. Canad. J. Math. 68 (2016), 445-462.

[21] M. Takahashi, On completely integrable first order ordinary differential equations, Proceedings of the Australian-Japanese Workshop on Real and Complex singularities. (2007), 388-418.

[22] M. Takahashi, Envelopes of Legendre curves in the unit tangent bundle over the Euclidean plane. Results Math. 71 (2017), 1473-1489.

[23] R. Thom, Sur la thorie des enveloppes. J. Math. Pures Appl. (9) 41 (1962), 177-192.

Masatomo Takahashi,

Muroran Institute of Technology, Muroran 050-8585, Japan,

E-mail address: masatomo@mmm.muroran-it.ac.jp 